\title{
Quantifying Vicious Circle Dynamics: The PEDA Model for Population, Environment, Development and Agriculture in African Countries
}

Wolfgang Lutz (lutz@iiasa.ac.at)

Sergei Scherbov (s.scherbov@frw.rug.nl)

\section{Approved by}

Gordon J. MacDonald (macdon@iiasa.ac.at)

Director

September 20, 1999 


\section{Contents}

Introduction: Population-Environment Models ................................................. 1

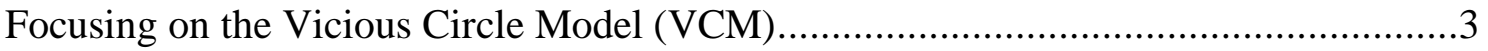

The Population-Based Approach to Population-Environment Modeling .......................5

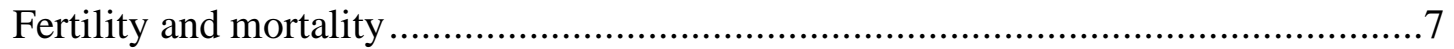

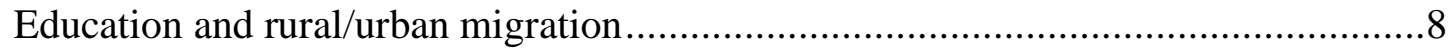

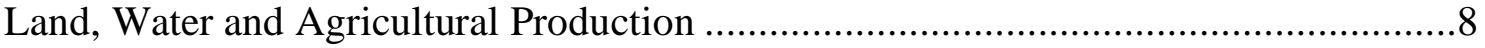

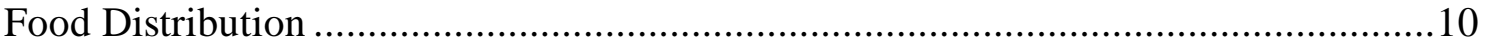

Operationalization and Sample Application to Burkina Faso ..................................12

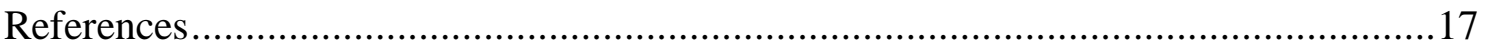




\section{Abstract}

This paper develops a quantitative simulation model linking population parameters and education to land degradation, food production and distribution, and resulting in the proportion of the population which is food insecure. This model is inspired by the Vicious Circle Model of Dasgupta and others, but can be applied more generally to interactions between these variables. The model chooses a population-based approach which groups individuals into eight categories as defined by rural/urban place of residence, literacy status and food security status. Using the tools of multi-state population projections, each group is simulated by age and sex. The model links this population module to an agricultural production function and a food distribution function which considers the fact that not all people have equal access to the food produced. This model has been applied to several African countries. Here it is illustrated with an application to Burkina Faso. 


\section{Acknowledgments}

This paper was presented at the Workshop on Population-Development-Environment, held at the Max Planck Institute for Demographic Research, Rostock, Germany, 12-13 August 1999. 


\section{About the Authors}

Dr. Wolfgang Lutz is the Leader of the Population Project at IIASA.

Dr. Sergei Scherbov is from the University of Groningen, Population Research Centre, NL-9700 AV Groningen, the Netherlands. 


\title{
Quantifying Vicious Circle Dynamics: The PEDA Model for Population, Environment, Development and Agriculture in African Countries
}

\author{
Wolfgang Lutz and Sergei Scherbov
}

\section{Introduction: Population-Environment Models}

Broadly speaking, in the field of population-environment models we can distinguish between two kinds of approaches: 1) comprehensive models that try to assess the full range of population-environment interactions for a specific region, and 2) other models that limit the focus to specific assumed chains of causation and therefore tend to be more focused and theory-driven. Both approaches can contribute to the better understanding of this complex field of studies. Both have their strengths and weaknesses. The more comprehensive (holistic) approach (1), which tries to evaluate all relevant factors, can help us to better understand the relative contribution of specific factors to the full picture. The series of PDE (Population-Development-Environment) case studies conducted by IIASA in different parts of the world are a good example for such comprehensive studies, which try to incorporate all relevant factors. For example, the recent applications to Namibia and Botswana which originally planned to focus primarily on population growth and water scarcity, had to be modified because the HIV/AIDS pandemic turned out to be of paramount importance for the future. Since around one-third of the population of these two countries is estimated to be HIV positive, IIASA has decided to dedicate a big share of the effort to HIV modeling. The PEDA model presented in this paper follows the other, substantively more focused, strategy (2). It attempts to quantify one specific causal path which actually is an assumed loop or circle that follows a clearly-defined theoretical model. It is restricted to portraying factors that are relevant to that specific mechanism, leaving out all others. This approach is more in line with the tradition of economic modeling that tends to make ceteris paribus assumptions on all factors that are not directly relevant to the hypothesis studied, even though such factors may be very significant for the future of a country under a more comprehensive approach. For planning purposes and sciencepolicy interactions, both the more comprehensive and the focused approaches have their virtues and shortcomings. In an ideal world a comprehensive super-model may incorporate several focused models, but this is difficult to achieve and may, indeed, suffer from some of the well-known shortages of mammoth models.

Having dwelt on the difference, it is also worth noting that both approaches, i.e., the IIASA-PDE approach and PEDA, have important features in common, namely an emphasis on (a) interdisciplinary scientific analysis and projection, and (b) sciencepolicy communication. 
(a) Interdisciplinary scientific analysis and projection lies at the heart of both approaches. In dealing with cross-cutting issues such as the effects of education on fertility and population structure and in turn on agricultural productivity and food security, the model necessarily needs to refer to the state of scientific analysis in a number of different disciplines, ranging from demography to economics, agricultural sciences, land use analysis and even water engineering. By putting information down in quantitative terms and specifying the specific quantitative inter-dependencies such a computer model can also help to contribute to overcome traditional disciplinary boundaries that have been characterized by specific research paradigms and approaches. Such models can contribute to improved communication between the disciplines by inviting scientists from the different disciplines to add to the model the specific structure and data they consider appropriate without losing the interaction with the other segments of the model. Specific empirical case studies (at the national or sub-national level) seem to be the right strategy to advance this goal and they are also the most useful under a policy perspective when the model is used to produce alternative projections under alternative policy-relevant scenarios.

(b) Science-policy communication: Similar to science being broken down into different disciplines, government policies tend to be compartmentalized according to the competencies of different ministries. This works well for some areas where the issues are limited in scope and require specialized treatment, but it does not work well for cross-cutting problems. Issues such as food security have to do with population, the skills of the labor force, agricultural production technologies and environmental issues such as soil quality and water availability. These diverse aspects do not fall into the responsibility of any one ministry in any country of the world. For this reason new ways need to be found to have interministerial connections to reflect the fact that in the real world things are also interconnected. An inter-sectoral model such as PEDA can help to demonstrate the usefulness and even the necessity for several ministries to work together on these issues. Furthermore, specific quantitative figures showing the outcomes of alternative policy choices over the coming decades are an efficient means of communication between scientists and policy makers. When using such models scientists do not only provide policy makers with vague opinions or unproven recommendations, but they can clearly and quantitatively demonstrate what alternative outcomes are to be expected, given of course that the specific assumptions of the model are accepted. But if the assumptions seem inappropriate, they can be changed and the new results can be compared to the old ones.

PEDA has been commissioned by the United Nations Economic Commission for Africa (UN-ECA) as an advocacy tool at the national level. In this it should compare with the widely used RAPID models but with a different focus and - this was the precondition of the authors' participation - a strong scientific foundation. It is meant to do advocacy not for any specific policy but for policies to take account of the strong existing nexus between population, environment and agricultural development. By the end of 1999 PEDA will have been initialized for six African countries (Burkina Faso, Cameroon, Madagascar, Mali, Uganda, and Zambia). It is planned to be applied to several more African countries in which food insecurity is a serious issue. 


\section{Focusing on the Vicious Circle Model (VCM)}

In recent years a theoretical model, often labeled the "vicious circle model," has become a very influential paradigm for describing the interactions between population growth, low status of women and children, environmental degradation and food insecurity. It is essentially an extension of the basic neoclassical economic model which adds a poverty trap based not on the macroeconomic reasoning of Malthus, but on microeconomic effects at the household and community level (O'Neill et al., 1999). This model is based on the assumption that high fertility, poverty, low education and low status of women and children are bound up in a web of interactions with environmental degradation and declining food production, in such a way that stress from one of these sources can trap certain rural societies, especially those living in marginal lands, into a vicious circle of increasingly destructive responses.

One possible illustration of this assumed pernicious interaction between high fertility and environmental degradation is the parable of the firewood (Nerlove, 1991). The gathering of firewood in many countries still relies heavily on children, and takes up an important part of their time. More children can collect more firewood for a family, but more firewood collection means less firewood near the village, increasing environmental degradation and longer ways to go for firewood. This takes even more of the children's time, which not only deprives them of educational opportunities but also tends to reinforce fertility. At least in the short run, the vicious circle argument is that higher fertility may be advantageous for a household in order to cope with the consequences of environmental degradation.

Dasgupta (1993) presents this argument in a more generalized form. The condition of poverty and illiteracy of the households concerned prevents substitution of alternative fuel sources or alternative livelihoods. A gender dimension is being added through the fact that the low status of women and girls also devalues the increasing amount of time and effort that they must devote to daily fuelwood gathering (Agarwal, 1994; Sen, 1994). The education of girls is blocked because girls are kept at home to help their mothers. The result is faster population growth, further degradation of the renewable resource base, increasing food insecurity, stagnating education levels, and yet a further erosion of women's status.

Leaving aside its actual empirical relevance, this vicious circle reasoning presents an important contribution to theory: it provides a unified framework for fertility, poverty, low female status and environmental degradation. It is politically attractive because it explicitly addresses equity concerns. Its multi-dimensional structure helps to view different possible interventions in, e.g., reproductive health, education, environmental conservation and agricultural efficiency in a unifying context rather than in isolation from each other. Each of the interventions may, under certain conditions, contribute to breaking up the vicious circle, but a comprehensive strategy viewing all these aspects together and recognizing their interdependencies is likely to be more successful.

Because the economic reasoning in the VCM largely operates at the household level, empirical studies on the issue have largely been confined to that level. But if the assumed mechanisms are at work, one can also expect significant macro-level consequences. In Africa these macro-level interactions between population, education, environment and food security seem to be particularly relevant and have recently been made one of the top priorities of the UN-ECA. A recent paper issued by the UN-ECA (1998), "Addressing the Food Security/Population/Environment Nexus in Africa," says: 
To alleviate poverty, the expected paramount goal of the African countries is to achieve policy objectives of sustainable development in the endeavour to harmonize population growth with utilization and exploitation of natural resources through re-direction, reorientation of research and development, and institutional changes. The first priority in this harmonization is to address challenges posed by the negative synergy arising from rapid population growth, food insecurity and environmental degradation. Despite repeated pledges by African leaders to address these issues, the rhetoric has frequently outrun performance.

The paper goes on to state that these immense challenges posed by poverty, rapid population growth, gender inequity, environmental degradation and food insecurity that traditionally have been addressed in isolation by the international and national policy communities need to be addressed together as a "web of strong and intricate interlinkages" that will shape the future living conditions of the African population. In making this point explicit reference to the VCM is made.

Figure 1. Basic structure of the PEDA model.

\section{PEDA AFRICA}

A Model Linking Population, Food Security and the Environment

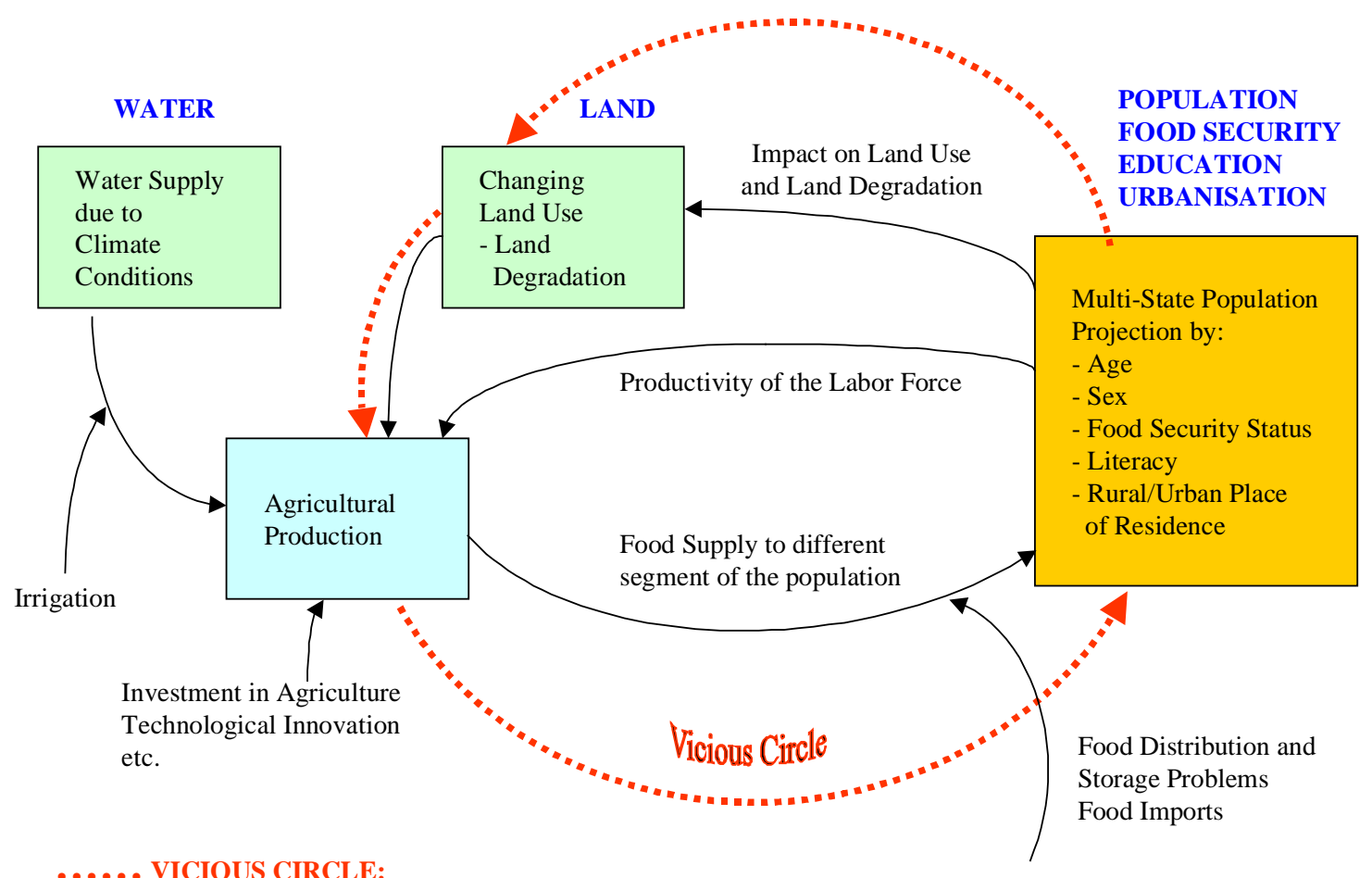

High population growth of the rural food insecure population will contribute to degrade the marginal lands. This decreases agricultural production which in turn still increases the number of food insecure persons.

In the spirit of this approach PEDA has been developed as a model that among other things can help quantify assumed vicious circle dynamics at the level of individual African countries. The PEDA model (as summarized in Figure 1) can be set up in a way 
that rapid population growth due to high fertility of the illiterate, food-insecure population in marginal rural areas ${ }^{1}$ contributes to further degradation of the land, thus lowering agricultural production and further increasing the number of food-insecure. If not broken this vicious circle would lead to ever increasing land degradation and increases in the food-insecure population. The circle can be broken, however, through several possible interventions in the field of food production, food distribution, education, environmental protection and population dynamics. Such a quantitative model can help policy makers and other users to (a) view these interconnected aspects, and (b) think in terms of alternative outcomes of alternative policy scenarios.

In its basic structure the PEDA model is rather flexible and not confined to quantification of vicious circle dynamics. Without activating the feedback mechanism of food supply and distribution, it can be used as a powerful multi-state population projection tool. But even in its feedback dynamics, the model can accommodate possible other theoretical positions, for example, if a user does not accept the vicious circle reasoning, but rather wants to run the model with the Boserupian assumption that high growth of the rural (uneducated) population actually increases per capita agricultural production. In this case, the model is flexible enough to run such scenarios after slight modifications in the software.

PEDA is different from most economic models in that it uses a population-based approach as will be explained in the following section. As compared to the IIASA-PDE models, it further expands on the idea of population-based analysis, i.e., breaking the population into several subgroups by age, sex, place, of residence, education and food security status and linking these population variables directly to physical variables such as water, land and calories available without going through much economic modeling.

\section{The Population-Based Approach to Population-Environment Modeling}

The population-based approach views human beings and their characteristics (such as age, sex, education, health, food security status, place of residence, etc.) as agents of social, economic, cultural and environmental change as well as agents who are at risk for suffering from repercussions of these changes, but also benefiting from positive implications. In this sense the human population is seen as a driving force of these changes and is affected by the outcomes and consequences of these changes. Economics, if it comes into the picture, e.g., through the importance of markets in distributing goods, plays only an intermediate role and is not seen as an end in itself or the primary object of modeling. In this the population-based approach differs from much of the development-economics literature.

The population-based approach does not assume that population growth or other demographic changes are necessarily the most important factors in shaping our future. It must not be misunderstood in the sense of a narrow view in which only demography matters. Instead the phenomena that we want to model are studied in terms of different characteristics that can be directly attached and (at least theoretically) measured with individual members of the population. Characteristics such as age, sex, literacy, place of

\footnotetext{
${ }^{1}$ Unlike some of the theoretical models, PEDA does not assume that fertility increases due to land degradation. Fertility is an exogenous variable here, for which scenarios can be set by the user for each of the eight sub-populations.
} 
residence and even nutritional status can be assessed at the individual level. The sum over these individual characteristics makes up the distribution in the total population. These individual characteristics are different from other frequently-used indicators such as the GNP per capita that cannot be directly measured with individuals. Although GNP per capita is suggestive of the average amount of money that an individual has in his/her pocket, it cannot be directly measured but rather results from a certain way of aggregate level national accounting with various conceptual and measurement problems. Although many of the powerful quantitative economic tools cannot be applied due to this choice of approach, other very powerful but less well known tools of demographic analysis and projection can be applied. The tools of multi-state population analysis allow for the projection of the population by several characteristics (such as age, sex, education and place of residence) at the same time. Multi-state projection groups all individuals of a given population into different sub-populations which are then projected into the future, while in each time interval, people can also move from one sub-population to another (e.g., from rural to urban or illiterate to literate for each sex and age group).

Figure 2. PEDA-population segment: A multi-state model by place of residence, education and food security status.

\section{PEDA AFRICA}

Segment on Population, Food Security, Education, and Place of Residence

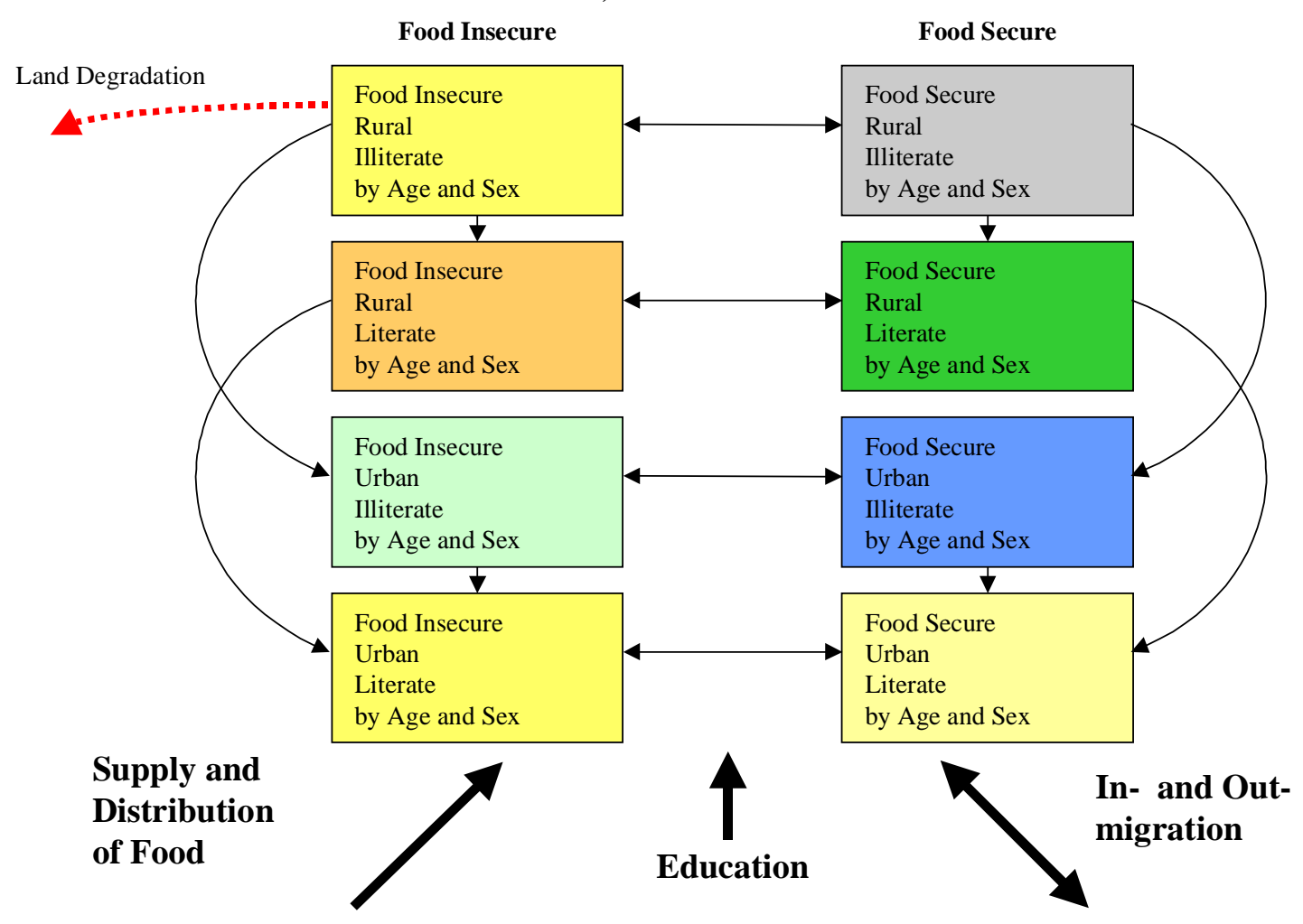


As shown in Figure 2, in PEDA the population of a country under consideration is broken down into eight sub-groups according to urban/rural place of residence, education and food security status. Place of residence and food security status are two dimensions which are core elements of the vicious circle reasoning as specified in this setting. Education, or more precisely literacy status, has been introduced into the model as one of the assumed key sources of population heterogeneity, which is related to both agricultural production and land degradation. Significant educational fertility differentials give the explicit consideration of education in the model a strong rationale. There is abundant literature on the significance of literacy in population-developmentenvironment interactions (see, e.g., Lutz, 1994). The potential of explicitly including education as a demographic dimension in multi-state population projection models has recently been evaluated (see Lutz et al., 1999) and is strongly recommended in the case of educational fertility (or other behavioral) differentials.

Each of these eight sub-groups further subdivides the population by age and sex, i.e., every one of the eight groups has its own age pyramid. During each one-year simulation step, a person will move up the age pyramid by one year within the same sub-group, or move to another sub-group while aging by one year. The movements between groups that are possible within each step are shown by arrows in Figure 2. For education and rural/urban migration, the model is hierarchical, i.e., people can only move in one direction, from lower to higher education and from rural areas to urban areas. Movement between food security states can happen in both directions, depending on the food conditions in the relevant year.

\section{Fertility and mortality}

In addition to the movements between sub-groups, each of the groups also experiences the vital events of births and deaths.

Different sets of age-specific fertility rates are applied to the female populations in each of the sub-groups. The data for the starting year are based on empirical information about differential fertility rates by education and urban/rural place of residence. Fertility differentials by food security status typically need to be assumed because of the absence of empirical information. For the future years, fertility within each sub-group can either be held constant or be changed according to an assumed linear trend.

The births generated through the application of those age-specific fertility rates will be put at the bottom of the age pyramids (after applying a sex ratio at birth) in the food security or residential state of the mother, i.e., it is assumed that children of an urban mother are also urban and children of a food-insecure mother are also food-insecure. With respect to education, the children may end up in a different sub-group than their mothers, because babies cannot yet be literate. In other words, all children of educated mothers are put into the uneducated category for their initial years and can then only move to the educated groups according to the educational transition rates assumed in the specific scenario.

Age- and sex-specific mortality rates can also be set up independently for the different groups. This can have important implications for population dynamics since the food-insecure groups can be expected to have much higher mortality rates than the food-secure groups. Unfortunately, since almost no empirical information exists on these mortality differentials, most of the differentials have to be based on assumptions. 
Sensitivity analysis can then demonstrate the implications of different assumptions. Of course, special survey information on this could provide the necessary empirical data.

\section{Education and rural/urban migration}

As indicated above, it is assumed that persons can only move from the illiterate to the literate state, but if considered important, secondary illiteracy can be incorporated relatively easily. For simplicity, it is assumed that all education takes place in childhood, but again, adult literacy campaigns can be incorporated, if necessary.

In the model the transition to literacy can be defined in terms of the total educational transition rate, which defines the proportion of each cohort of girls or boys that will become literate.

In a similar fashion, the level of rural to urban migration can be defined through the total migration rate, giving the proportion of each rural cohort to move to urban areas. The only difference to education is that the migration is assumed to be less concentrated in a specific age group, but is spread over a broader age range according to typical agespecific migration patterns.

International migration has not yet been specified as a scenario variable in this prototype application. For simplicity, countries are being considered as closed populations. But it is not difficult to explicitly include international migration to and from the specific sub-populations by age and sex. Theoretically, if considered appropriate, the PEDA models of neighboring countries could also be linked through international migration and considered simultaneously.

\section{Land, Water and Agricultural Production}

As indicated in Figure 1, the population module, i.e., the population by age and sex in the eight defined categories and for each year in time, affects the total agricultural production in two different ways. The productivity of the rural labor force as measured by the proportion literate of the rural population of productive age will directly enter the agricultural production function as discussed below. The other chain of causation is a direct reflection of the vicious circle reasoning: the factor land is degraded as a function of the increase in the number of people in the rural, food-insecure and illiterate category. In the current version of PEDA this impact is operationalized in the following manner: the total amount of high quality agricultural land enters the production function in an index form which is assumed to combine quantity and quality aspects. The higher the increase (as compared to the starting conditions) in this critical group of foodinsecure, rural, illiterate population, the more this land factor will decline. The user can set a scenario variable, the land degradation impact factor, that determines to which degree a certain percentage increase in this critical population impacts on the land index.

Water is another important environmental factor for food security and agricultural production. No life can exist without water. And certainly no economic or human development is possible without water. Yet hardly ever is water explicitly included in models of population and development. Even most agricultural production models such as the one chosen here (see Hayami and Ruttan, 1971) do not include water as a variable in their production functions. The natural availability of water tended to be considered 
self-evident and not worth any special consideration. This view, however, has changed over the recent years, especially in the context of trying to understand the possible consequences of global environmental change on agriculture.

Water only becomes a problem for food production if the demand (including household consumption and industrial consumption in addition to agricultural demand) exceeds the supply. Water supply, however, can vary greatly according to short- and long-term natural fluctuations in rainfall. It is greatly influenced by the surface structure, the pattern of river basins and groundwater systems, and it can be strongly influenced by human engineering (dams, irrigation, etc.). Because water is so important for sustainable development and follows complex non-linear dynamics, the PDE models developed by IIASA all have water modules that tend to be at least as complex as the population modules. For PEDA, however, water modeling is not a prime focus. In the vicious circle model, water only matters as one additional exogenous factor that can have impacts on the total food production, especially with respect to drought on the one hand, and irrigation efforts on the other. For this reason PEDA treats water as an index variable (with 1.0 reflecting the average conditions around the starting year) that can be enhanced through irrigation or natural changes and diminished through declines on water supply.

The total agricultural production in one year, measured in total calories produced, will then be a result of the input in terms of human labor force by different educational levels, water, land, and technological inputs such as fertilizers, mechanization, etc. Those additional inputs will also be treated in terms of externally-defined scenarios because these factors are not assumed to depend directly on other variables of the PEDA model. (If a user, however, wants to make, e.g., the rate of new agricultural investment dependent on population growth in either a positive or negative way, it is not difficult to do so and study the alternative results.) The specific agricultural production function used here has been derived from an internationally highly renown book in the field (Hayami and Ruttan, 1971). This production function will then result in a certain total calorie production by the end of the year.

Many agricultural production functions exist, but most of them surprisingly do not consider the labor force and the skills of the labor force as a production factor, but largely focus on physical and financial inputs. A notable exception is the above mentioned book by Hayami and Ruttan (1971). Based on pooled data sets of time series in most countries in the world, they estimate large numbers of Cobb-Douglas type production functions with different combinations of input factors and for different groups of countries. The one equation that seemed most appropriate for the PEDA Africa model is the one giving a Principal Components Regression for developing countries, including education variables. Agricultural output in our case is measured in terms of total calorie production (in index form).

The elasticities and the specific variable definitions in PEDA are taken from Hayami and Ruttan (1971, p. 145, Q 19):

$.534 *$ Rural Labor Force (specified here as total rural adult population aged 15-60, calculated from combining the appropriate age groups in all four rural sub-groups

$.088 *$ Total Agricultural Land (can be modified through land degradation or the clearing of new land as discussed)

$.162 *$ Fertilizer Use (will be treated as an exogenous scenario variable) 
$.072 *$ Tractors Available (will be treated as an exogenous scenario variable called more broadly "mechanization")

$.276 *$ Literacy (specified here as the proportion literate of the total rural population aged 10-45, calculated by combining both the food-secure and the food-insecure rural literate sub-populations)

$.158 *$ Technical Education (still treated here as an exogenous scenario variable, may later be related to the educational efforts parameter)

All these input variables to agricultural production are considered here on a relative scale, i.e., they are set to equal 1.0 in the starting year, and then change over time as it results from the other sectors of the model for the endogenous production factors or as defined in the scenario setting for the exogenous variables. (For example, an assumed increase in fertilizer input of 20 percent by 2003 would mean that the variable is set to gradually increase to 1.2 by that year.)

Unfortunately, in reality, not all the production will be consumed by individuals to satisfy their food needs. Some calories will be lost during the treatment of the food, others will be lost during transport and some will be lost due to inadequate storage. Of the food that will actually reach people for consumption, a certain fraction will go to urban areas and another to rural areas. All these factors can be assumed in PEDA as scenario variables specific for a country and can be changed over time, or alternative starting values can be assumed. More specifically, in the model there are three different scenario variables that the user can set: loss in transport and storage, food import/export, and an urban bias factor. The latter determines to what degree the total available food should be disproportionally distributed between urban and rural areas. If this factor is 1 , then food will be distributed according to the population size of urban and rural areas. Within these areas, however not everybody will receive an equal amount of food. Reality shows that there are gross inequalities in access to food and therefore, PEDA has an explicit food distribution module.

\section{Food Distribution}

Even when the total amount of food reaching the (urban and rural total) population would be theoretically sufficient to provide the necessary minimum diet for everybody, in practice the distribution of food is unequal because some persons do have more purchasing power than others or have privileged access to food by other means. This will result in the fact that some people remain food-insecure even when the average total amount of food reaching the population is above the minimum.

There is abundant empirical evidence, backed up by theoretical considerations, clearly showing that the distribution of food is at least as important as the total production of food in explaining food insecurity. Especially the path-breaking work of Amartya Sen (1994) demonstrated that some of the worst famines occurred under conditions in which theoretically there would have been enough food for everybody if the distribution had been appropriate. For this reason it is evident that a model focusing on food security without paying attention to the distributional aspects would be incomplete, if not misleading. The main problem with considering such distributions, however, lies in the fact that hardly any empirical data exist on distributive mechanisms in the countries of Africa today, and that theoretical distributions are hardly appropriate because conditions tend to vary significantly from one country to another. As a solution to this problem, in PEDA we chose to approximate the food distribution function 
through an income distribution function, which exists for a number of African countries based on household income surveys. This allocation of food to urban and rural populations and the food distribution within these populations then determines the new sizes of the food-secure and food-insecure sub-populations in the following year.

Figure 3. Food distribution function.

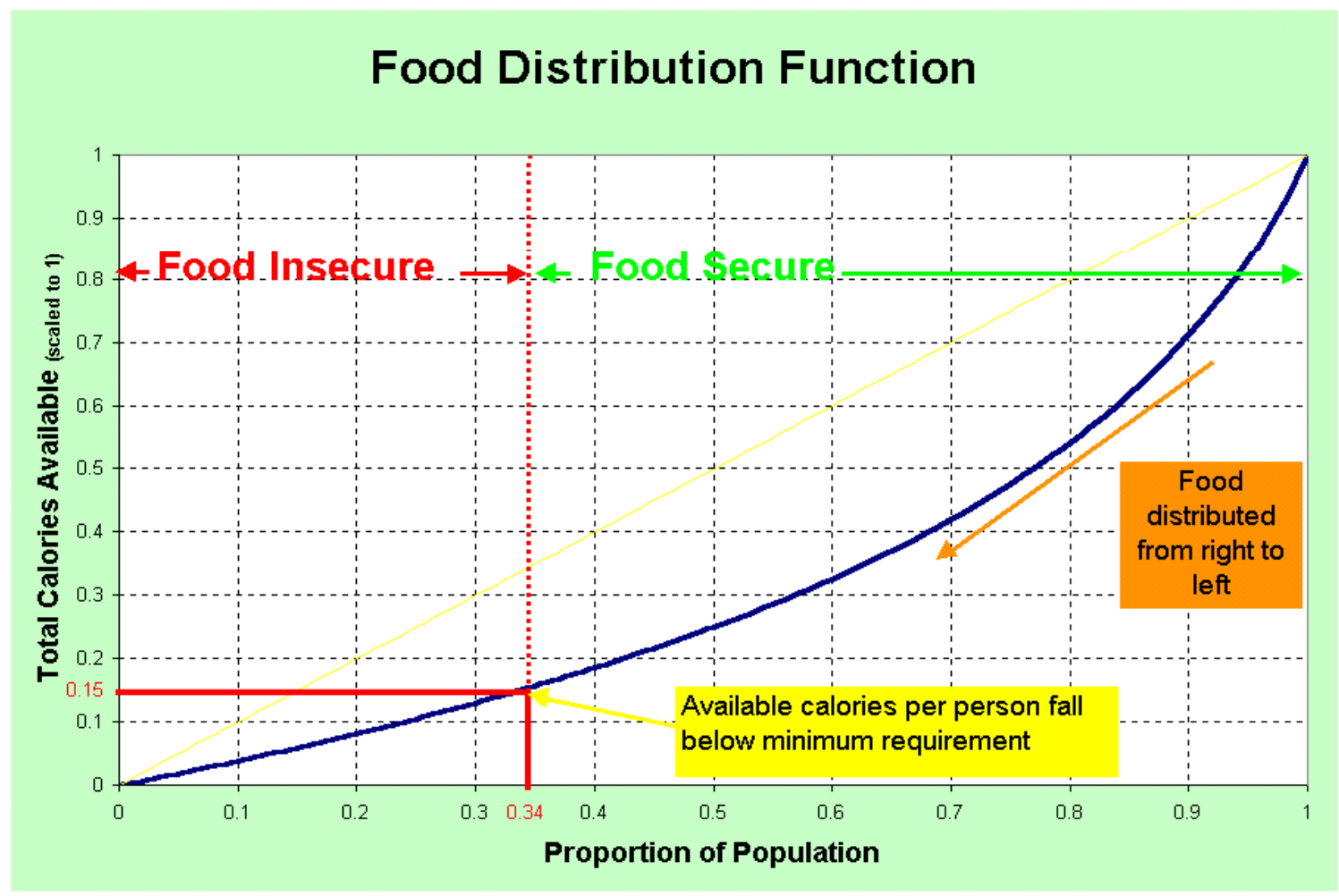

Figure 3 shows such a food distribution function that is applied after allocating the total available food to urban and rural populations (according to an exogenously-defined "urban bias" variable). This figure shows a Lorenz curve with the cumulated proportion of the population on one axis and the cumulated calories available for distribution on the other. The available food is then distributed from left to right along the black curve. The given curve indicates that in this case, the first (most privileged) 10 percent of the population use 30 percent of the available food. Going further down the curve, about 23 percent of the population use half of the food, and half of the population uses 75 percent of the food. The borderline between the food-secure and the food-insecure population is then established by applying an externally-defined minimum calorie requirement per person. At the point where the remaining food supply falls below the minimum requirement times the remaining population, the border line for the population considered to be food-insecure is established. Over time the proportions food-insecure may change as a consequence of changes in the calories available for distribution or possible changes in the assumed food distribution function. 


\section{Operationalization and Sample Application to Burkina Faso}

PEDA needs empirical data primarily for the starting conditions, for the transition rates between the different population groups, and for the factors associated with food production and food distribution. Since data sources for many African countries are limited, some of these data need to be based on estimations and assumptions. For the starting year one must have the distribution of the population by age: in 100 single-year age groups, sex (female/male), food security status (food-secure/food-insecure as defined below), literacy (literate/illiterate according to the UNESCO definition) and urban/rural place of residence (according to the national definition of a town). If the information is not readily available, one must estimate some of the distributions, as will be discussed below.

Before the model can be applied to a new country, it needs to be initialized. If all data would be readily available by single years of age, this would be a relatively straightforward process of entering data. Unfortunately, only a few countries in the world have this kind of data available, and none of them are in the African region. Hence, the process of initializing the model for African countries requires quite elaborate estimation techniques whose specific nature will vary from case to case, depending on the kind of empirical information available. In most cases, this requires special skills in mathematical demography and the programming of macros for spreadsheets. For this reason the process of initialization will not be described here. For the following description we assume that a country application has already been initialized.

The model is based on Excel and will run under Windows 95, 98, NT, 2000. It should run on any standard PC (given that all the necessary libraries have been installed) but the performance will depend on the speed of the hardware.

The model runs in single years of time. In the first year the people that are members of the eight different sub-groups will impact in two independent ways on the rest of the model. This is indicated in Figure 1 of the vicious circle by the two solid arrows leaving the population box on the right. Together with the other input factors these population factors will then result in a certain total calorie supply in the following year $(t+1)$. After adjustments for loss and import/exports in that year, the above-described food distribution function will then determine the proportions considered food secure and insecure in urban and rural areas. Together with the demographic transition rates (fertility, mortality, migration, education, etc.) applied between time $t$ and $t+1$ this food distribution will then result in the new distribution of the total population by age and sex over the eight states at time $t+1$. This population will then serve as input to the agricultural production at time $t+2$, and so on.

We choose Burkina Faso as an example of a country that has already been initialized. It is a country with high food insecurity, high illiteracy, very high fertility and land degradation problems, which are rather typical for the whole Sahel region. For this application all data have been derived from internationally available sources. Missing data had to be estimated using various indirect procedures. It is, of course, desirable to ultimately run the model with as much empirical data as possible. This needs to be collected in collaboration with local scientists. 
Figure 4. Distribution of the population in Burkina Faso over the eight sub-populations in 1996 (Fig. 4a) and in 2030 under the Constant Rates Scenario (Fig. 4b).
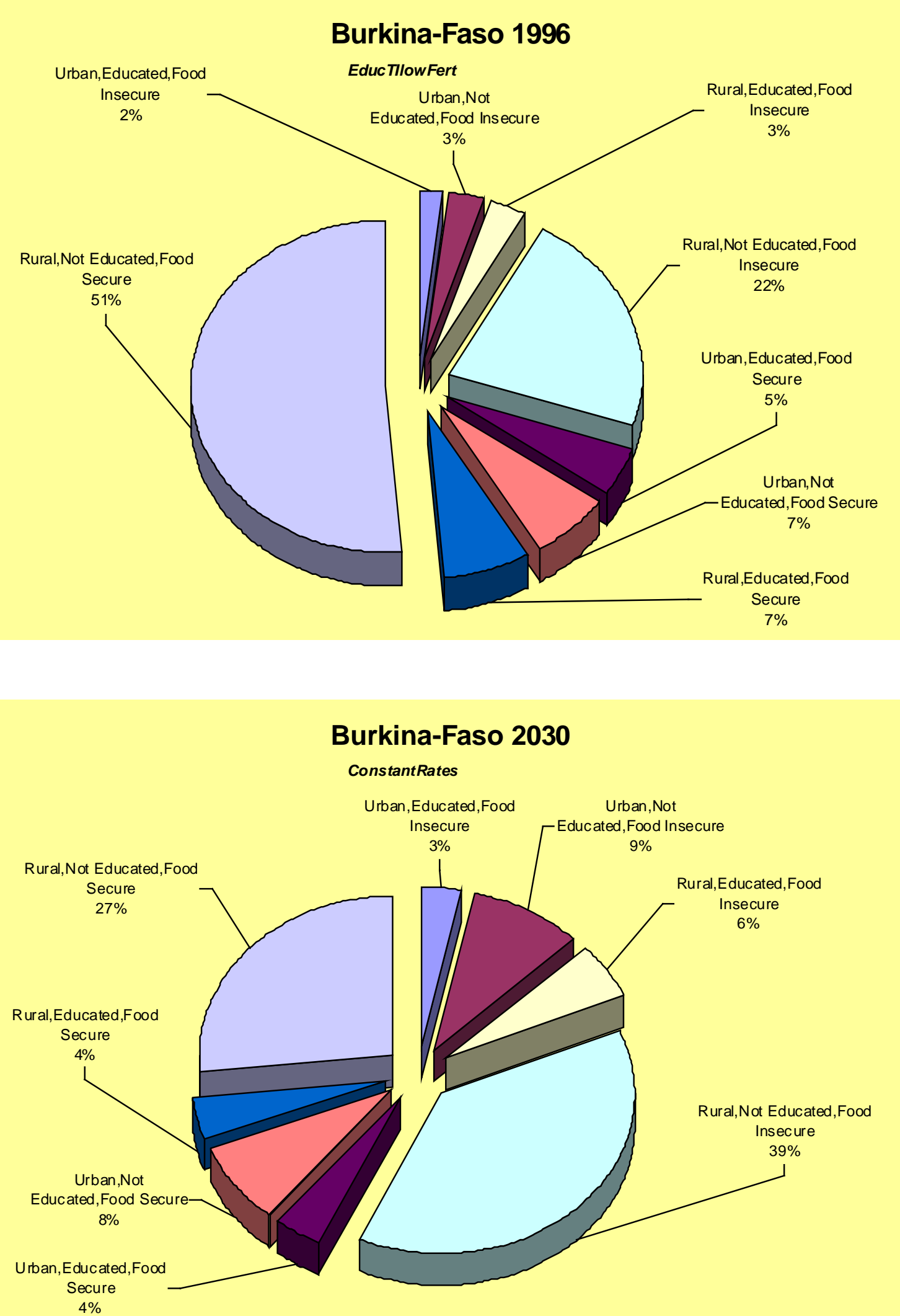
Figure 4a gives the initial distribution of the population of Burkina Faso in 1996 according to the eight sub-populations. We can see that in 1996 the majority of the population belongs to the rural/illiterate/food-secure sub-population. The second largest group comprising almost one-quarter of the population is the rural/illiterate/foodinsecure population. Of the other six sub-groups none contains more that $7 \%$ of the total population.

Current fertility levels in Burkina Faso are still very high. According to surveys, the rural illiterate population has a TFR of almost seven. The rural literate and the urban illiterate populations have rather similar fertility levels around five. Only the urban literate population has levels slightly below four. Because of a lack of empirical information about fertility by food security status, we assumed in these illustrative scenarios equal fertility levels for the food secure and insecure. These high fertility levels together with the fact that about three out of four women in Burkina Faso are rural and illiterate lets us expect very rapid population growth under the constant rates scenario.

The following four scenarios have been defined for illustration:

Constant Rates: All parameters (including fertility and school enrolment) remain at their 1996 levels.

Increased Technological Inputs: Fertilizer use, machinery and irrigation all increase by 3 percent per year (other parameters constant).

Strong Educational Efforts: 80 percent of all girls and 90 percent of all boys learn to read and write (other parameters constant).

Combining Increased Technological Inputs, Strong Educational Efforts and Fertility Decline: Levels decline to half of their 1996 values by 2030.

Figure 5. Scenario results for Burkina Faso for the total population (top lines) and for the food insecure population (bottom lines).

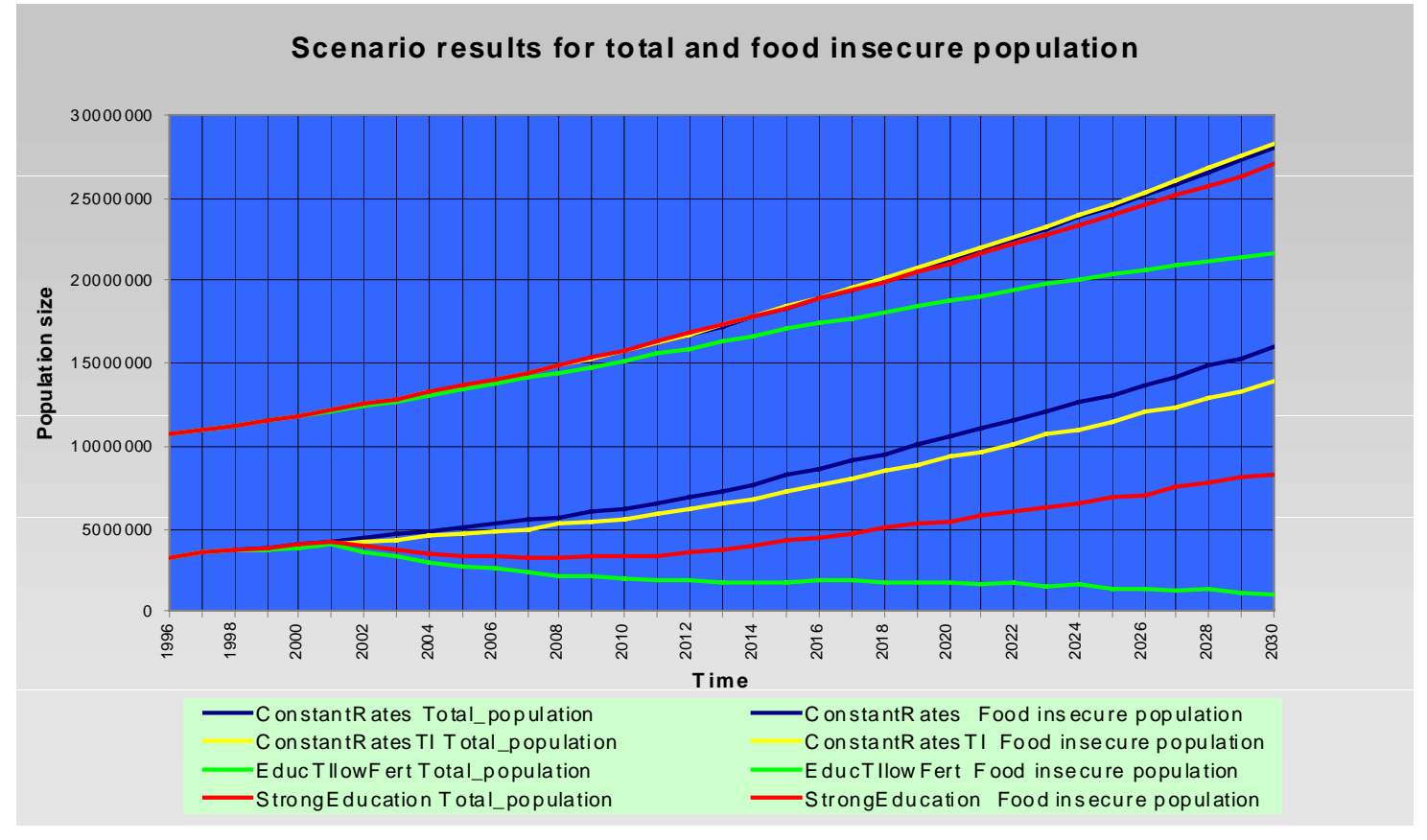


Figure 6. Scenario results for Burkina Faso. Proportion of the population that is food insecure.

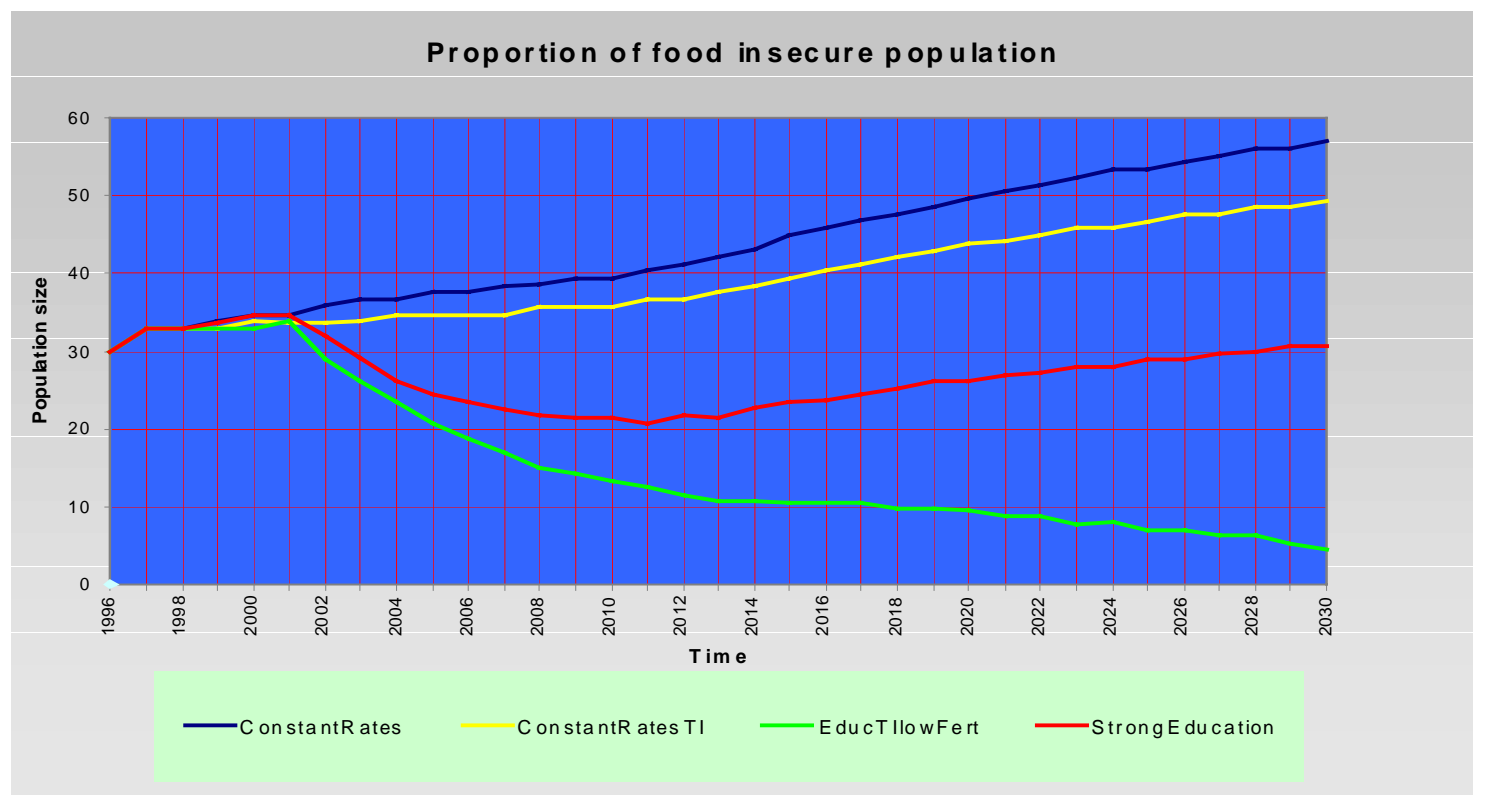

Figures 5 and 6 show the results for the four scenarios for Burkina Faso to the year 2030 giving the total population (all eight sub-groups combined) and the food-insecure population (all four food-insecure sub-groups combined) in two separate sets of lines (Figure 5) as well as the proportion food-insecure (Figure 6).

The constant rates scenario shows that over these 35 years the total population would increase by a factor of almost three. The food insecure population grows even more rapidly under this scenario with the proportion food insecure increasing from around 30 percent to more than 50 percent in 2030. The distribution over all eight states in 2030 under this scenario is depicted in Figure 4b.

Adding the increased technological inputs to otherwise constant parameters does not affect the total population size but has a visible impact on reducing food insecurity which, under this scenario, would not reach 50 percent by 2030 .

Adding the educational efforts to otherwise constant parameters (and no increased technological inputs) already has a visible impact on reducing total population size (through lower fertility of educated women) and also shows a very sizeable effect on reducing food insecurity (through the additional effect of higher productivity).

It is interesting to note that the effect of increased educational efforts only starts to become visible with a certain time lag, because education is concentrated in the younger ages that will only later enter the reproductive ages and the labor force. Hence in the very short run increased technological input has a great immediate effect, but in the longer run the educational effect is by far more important (at least under the specific assumptions made in this example). Figure 6 also shows that the decline in food insecurity brought along by education alone is not sustainable. After 2010-15 the proportion food-insecure starts to increase again because the still very high rates of population growth will outweigh the improvements in agricultural production. 
Only the fourth scenario, combining the increased technological inputs with the education efforts and an assumed gradual halving of fertility rates by 2030 , will result in a sustainable decline in the proportion food-insecure and in a path of total population growth that would "only" result in a doubling of the population by 2030 .

Since all information in PEDA is structured by age and sex, the model is very appropriate for detailed analyses of gender-specific issues (this is not yet part of the standard output graphs but can be easily derived from the full data base itself). Figure 7 shows the consequences of the four scenarios for the number of food-insecure women above age 60 in Burkina Faso. The number of women in this vulnerable group will increase very strongly (by a factor of more than five) under the constant rates and the technological inputs only scenarios. The educational efforts only scenario results in some stabilization over the next 20 years, which later will be overwhelmed by the impacts of high population growth due to constant fertility within each sub-population. As for the general population only, the combined scenario, which includes fertility decline, will result in a sustainable decline of this specific group of elderly foodinsecure women.

Figure 7. Scenario results for Burkina Faso. Number of food insecure women over age 60 .

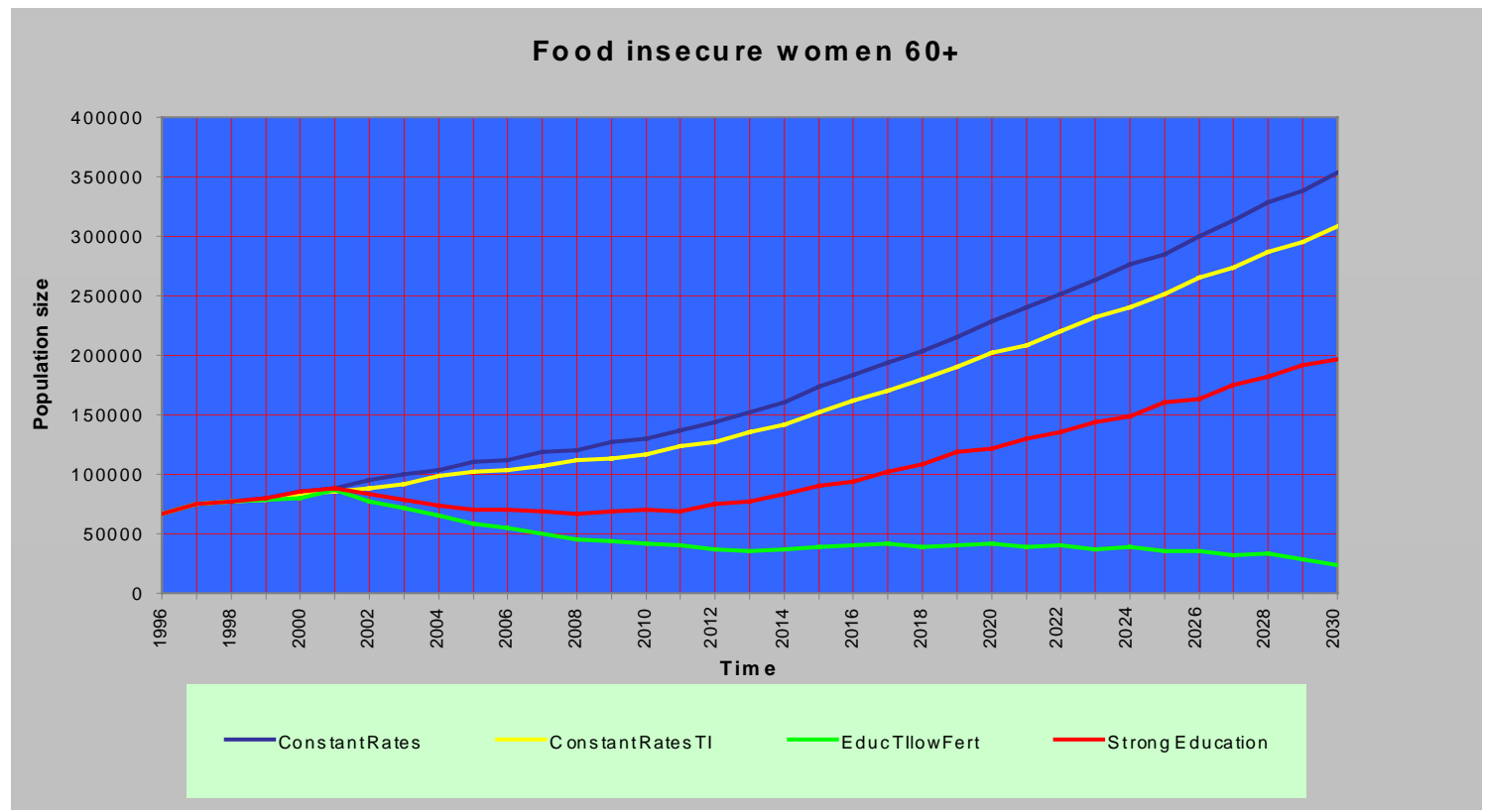

As stated above, these four illustrative scenarios result from four specific rather $a d$ hoc choices of model parameters. They provide some interesting stories and insights into the dynamics of the system. Other scenarios may tell other stories. The user of PEDA can calibrate innumerable different scenarios based on different parameter assumptions that he/she may find interesting or worth communicating to the policy makers. 
It is our hope that this effort of trying to quantify vicious circle dynamics for specific African countries presents a useful step in further advancing work on the model itself, empirical applications to real world conditions, interdisciplinary dialogue on population-environment interactions, and finally, science-policy dialogue on the importance of dealing with these interconnected issues in an integrated manner.

\section{References}

Agarwal, B. 1994. The gender and environment debate: Lessons from India. Pages 97124 in L. Arizpe, M.P. Stone, and D.C. Major, eds., Population and the Environment: Rethinking the Debate. Boulder, CO: Westview Press.

Dasgupta, P.S. 1993. An Inquiry into Well-Being and Destitution. Oxford, U.K.: Oxford University Press.

Hayami, Y. and V. Ruttan, 1971. Agricultural Development: An International Perspective. Baltimore, MD: Johns Hopkins University Press.

Lutz, W., Ed. 1994. Population-Development-Environment: Understanding their Interactions in Mauritius. Berlin: Springer Verlag.

Lutz, W., A. Goujon, and G. Doblhammer-Reiter. 1999. Demographic dimensions in forecasting: Adding education to age and sex. Pages 42-58 in W. Lutz, J.W. Vaupel, and D.A. Ahlburg, eds., Frontiers of Population Forecasting. A Supplement to Vol. 24, 1998, Population and Development Review. New York, NY: Population Council.

Nerlove, M. 1991. Population and the environment: A parable of firewood and other tales. American Journal of Agricultural Economics 73:1334-1347.

O’Neill, B.C., F.L. MacKellar, and W. Lutz. 1999. Population and Climate Change. To be published by Cambridge University Press, forthcoming.

Sen, A. 1994. Women, poverty, and population: Issues for the concerned environmentalist. Pages 67-86 in L. Arizpe, M.P. Stone, and D.C. Major, eds., Population and the Environment: Rethinking the Debate. Boulder, CO: Westview Press.

UN-ECA. 1998. Addressing the Food Security/Population/Environment Nexus in Africa. Internal Issues Paper 1998. Addis Ababa, Ethiopia: United Nations Economic Commission for Africa. 